\title{
影像组学研究进展及其在肝癌中的 临床应用
}

韩玉齐 ${ }^{1,2 \dagger}$, 魏靖伟 ${ }^{2 \dagger}$, 蒋涵羽 $^{3 \dagger}$, 牛猛 ${ }^{4}$, 刘 兵 $^{5}$, 付芳芳 ${ }^{6}$, 顾东 升 $^{2}$, 郝小涵 ${ }^{2,7}$, 李宏军 ${ }^{8^{*}}$, 宋涁 ${ }^{*}$, 田捷 $1,2,9,10,11^{*}$

1. 西安电子科技大学生命科学技术学院, 西安 710126 ;

2. 中国科学院自动化研究所, 分子影像重点实验室, 北京 100190 ;

3. 四川大学华西医院放射科, 成都 610041 ;

4. 中国医科大学第一附属医院介入放射科, 沈阳 110000 ;

5. 中国人民解放军总医院第一医学中心肝胆外科, 北京 100853 ;

6. 河南省人民医院, 郑州大学人民医院医学影像科, 郑州 450003;

7. 中国科学技术大学医学影像中心, 合肥 230027 ;

8. 首都医科大学附属北京佑安医院放射科, 北京 100069 ;

9. 分子影像北京市重点实验室, 北京 100190 ;

10. 中国科学院自动化研究所, 复杂系统管理与控制国家重点实验室, 北京 100190 ;

11. 北京航空航天大学医工交叉研究院, 大数据精准医疗高精尖创新中心, 北京 100191

$\dagger$ 同等贡献

* 联系人, E-mail: lihongjun00113@126.com; anicesong@vip.sina.com; tian@ieee.org

收稿日期：2019-12-17; 接受日期：2020-04-10; 网络版发表日期：2020-05-27

国家重点研究开发项目(批准号：2017YFC1308701，2017YFC1309100，2017YFA0205200，2016YFC0103001)、国家自然科学基金(批准号： 81527805, 81771924)、中国科学院前沿科学重点项目(批准号: QYZDJ-SSW-JSC005)和中国科学院科研仪器设备研制关键技术团队项目(批 准号: GJJSTD20170004)资助

摘要医学影像已经广泛用于医疗决策中, 如肿瘤诊断, 其包含了大量人眼无法识别的可以表达肿瘤异质性的 重要信息. 影像组学分析利用计算机技术识别医学影像中的这些信息, 将其转化为定量的影像特征, 结合相关的 临床问题, 为预后和临床决策提供一定的参考价值。影像组学的出现代表了医学成像的评估从一个描述性、定 性学科到预测性、定量学科的演变. 肝癌是我国常见的恶性肿瘤之一, 发病率和死亡率非常高. 影像组学已应用 于肝癌的诊断、治疗方法的选择和治疗疗效的评估三个不同阶段的不同临床问题中. 因此本文从影像组学的基 本原理与技术入手, 结合其在肝癌临床诊疗中的应用, 针对肝癌治疗过程中的不同问题分别介绍了影像组学的 应用。

关键词肝细胞癌, 影像组学, 诊断, 治疗方案选择, 疗效评估

引用格式: 韩玉齐, 魏靖伟, 蒋涵羽, 等. 影像组学研究进展及其在肝癌中的临床应用. 中国科学: 生命科学, 2020, 50: 1309-1320 Han Y Q, Wei J W, Jiang H Y, et al. Research progress and clinical application of radiomics inhepatocellular carcinoma (in Chinese). Sci Sin Vitae, 2020, 50: 1309-1320, doi: 10.1360/SSV-2019-0230 
临床成像方式，如电子计算机断层扫描(computed tomography, CT)、正电子发射断层扫描(positron emission computed tomography, PET)和磁共振成像(magnetic resonance imaging, MRI), 是现代肿瘤诊断和管理 的重要手段 ${ }^{[1,2]}$. 近年来, 人工智能领域的迅速发展使 得医学影像的角色正在从一种基础的诊断工具演变为 个性化精准医疗的重要工具 ${ }^{[3]}$. 在此背景下, 2012年荷 兰学者Lambin等人 ${ }^{[4]}$ 提出了影像组学(radiomics)的概 念. 作为一种新兴的技术手段, 影像组学将影像中潜在 的病理和生理学信息转化为可挖掘的高维定量影像特 征 ${ }^{[5]}$. 通过分析高维定量影像特征与临床数据和基因 数据间的关系, 实现肿瘤的诊断、分期分型和预后预 测等.

原发性肝癌是我国排名第4位的常见恶性肿瘤, 其 病理类型主要包括肝细胞癌(hepatocellular carcinoma, $\mathrm{HCC}$ )、肝内胆管癌(intrahepatic cholangiocarcinoma, ICC)和HCC-ICC混合型, 其中HCC大约占肝癌的 $85 \%$ $\sim 90 \%{ }^{[6,7]}$. 因此, 除特别说明外, 本文中“肝癌”指HCC. $\mathrm{HCC}$ 在全球恶性肿瘤发病率中排名第6位, 其中每年新 发病例和死亡病例有 $1 / 2$ 以上来自中国 ${ }^{[6]}$. 尽管肝癌的 诊断方案日益完善, 但肝癌的预后仍不理想, 是我国 第三位的癌症致死病因. 因此, 在我国开展肝癌的诊 疗及预后研究至关重要, 可以帮助医生为患者制定个 性化的治疗方案, 改善患者预后.

研究表明, 肝癌的生物学行为多样性影响患者的 生存状况, 而且肿瘤的异质性也可成为判断治疗效果 的重要线索 ${ }^{[8,9]}$. 因此, 肝癌的诊断和治疗趋势正根据 其生物学和基因组特征而发生变化, 而医学成像可以 作为探究这些特征的一种方法 ${ }^{[10 ~ 12]}$. 与常用的血清标 志物不同, 多数的影像学报告包含定性的或描述性的 特征, 这些特征容易受到放射科医生个人主观经验的 影响, 而这种观察者间的可变性仍是难以解决的问题. 影像组学技术对医学影像进行客观和定量的分析, 为 探究成像生物标志物提供了新的契机. 近年来, 影像 组学技术在肝癌中进行了多个方面的研究, 为个性化 的治疗决策制定提供了有力的帮助(表1). 因此, 本文 就影像组学技术及其在肝癌中的研究现状作一综述.

\section{1 影像组学流程}

影像组学是一种从医学影像(一般指CT、PET、
MRI或超声)中提取定量的放射学数据并探索其与临 床结果相关性的研究方法 ${ }^{[4,5,32]}$. 影像组学的处理流程 通常包括以下4个方面: (i) 影像采集; (ii) 感兴趣区 域(region of interest, ROI)的检测与分割(自动、半自动 或手动分割); (iii) 影像组学特征的提取; (iv) 数据挖 掘并构建模型以预测临床结果.

\section{1 医学影像采集}

在影像组学分析中利用标准化的成像协议消除不 必要的混杂变异因素至关重要 ${ }^{[32,33]}$. 然而, 非标准化成 像协议在临床工作中很常见. 因此, 为了满足影像组学 分析的再现性和可比性, 提供详细的成像协议是必 要的.

影像组学分析从影像的成像方式和ROI的选择入 手, 并最终实现临床目标的预测. 影像组学可以选择整 个肿瘤区域、肿瘤的亚区域或生长微环境(habitats)、 转移性病灶和正常组织等, 结合相应的治疗结果(如生 存期)进行分析. 对这些区域的分析能够得到对放射敏 感的表型特征, 从而对治疗策略产生影响.

\section{2 肿瘤区域分割}

ROI分割是影像组学分析的基础步骤, 其将原始 医学影像转化为可以进行特征提取的图像. 分割的首 要问题是考虑以何种方式进行, 以及影像组学分析对 不同分割方法的敏感性 ${ }^{[34]}$. 目前, 主要的ROI分割包括 自动分割、半自动分割和手动分割. 肿瘤图像的分割 算法常用的有國值分割、基于区域的图像分割、基于 边缘的图像分割和基于模糊理论的图像分割等. 肿瘤 的自动分割算法种类繁多, 而且对于不同情况如何选 择合适的算法以及不同分割算法对后续分析的影响如 何尚无统一标准. 目前, 影像组学分析中常用的方法是 由经验丰富的影像科医生手动地分割ROI(如ITKSNAP分割软件, www.itksnap.org). 但是, 人工分割易 受到主观因素影响, 需进行多重分割(multiple-segmentation) 以获得稳定特征, 如进行多个临床医生的分 割、添加噪声干扰、利用呼吸周期的不同阶段进行多 次分割等 ${ }^{[35]}$.

\section{3 特征提取和特征选择}

影像组学的本质是通过提取高通量的定量特征来 刻画ROI. 目前, 影像组学分析主要的特征提取方法是 
表 1 影像组学在HCC中的应用

Table 1 Application of radiomics in hepatocellular carcinoma

\begin{tabular}{|c|c|c|c|c|}
\hline 目的 & 样本量 & 特征类型 & 影像模态 & 引用 \\
\hline 肿瘤类型鉴别 & 177 & 深度学习 & Ultrasound & {$[13]$} \\
\hline 肿瘤类型鉴别 & 65 & 直方图 & MRI & {$[14]$} \\
\hline $\mathrm{HCC}$ 分级 & 170 & 直方图, 形状, 纹理 & MRI & [15] \\
\hline MVI预测 & 284 & 直方图, 形状, 纹理 & $\mathrm{CT}$ & {$[16]$} \\
\hline MVI预测 & 28 & 直方图, 形状, 纹理, 边缘 & $\mathrm{CT}$ & {$[17]$} \\
\hline MVI预测 & 482 & 直方图, 纹理 & Ultrasound & [18] \\
\hline MVI预测 & 160 & 直方图, 纹理, 小波 & MRI & [19] \\
\hline MVI预测 & 157 & 直方图, 形状, 纹理, 小波 & $\mathrm{CT}$ & {$[20]$} \\
\hline MVI预测 & 495 & 直方图, 形状, 纹理, 小波 & $\mathrm{CT}$ & {$[21]$} \\
\hline MVI预测 & 208 & 直方图, 形状, 纹理, 小波 & MRI & {$[22]$} \\
\hline 肝切和TACE选择 & 520 & 语义特征, 直方图, 形状, 纹理和小波特征 & $\mathrm{CT}$ & {$[23]$} \\
\hline 早期复发(1年) & 215 & 直方图, 纹理 & $\mathrm{CT}$ & {$[24]$} \\
\hline 早期复发(730天) & 50 & 纹理 & MRI & {$[25]$} \\
\hline 肝移植后复发预测 & 133 & 直方图, 形状, 纹理和小波特征 & $\mathrm{CT}$ & {$[26]$} \\
\hline 消融后复发 & 184 & 直方图, 形状, 纹理和小波特征 & $\mathrm{CT}$ & [27] \\
\hline 生存期 & 319 & 纹理 & $\mathrm{CT}$ & [28] \\
\hline 生存期 & 88 & 直方图, 形状, 纹理 & $\mathrm{CT}$ & [29] \\
\hline 生存期 & 138 & 直方图, 纹理 & $\mathrm{CT}$ & {$[30]$} \\
\hline 生存期 & 47 & 直方图, 纹理 & PET & {$[31]$} \\
\hline
\end{tabular}

将Aerts等人 ${ }^{[5]}$ 发表在Nature Communications 的方法改 进后加以利用. 特征提取是连接医学影像和临床分析 结果的桥梁, 特征类型可大致分为 4 种类型: (i ) 反映 肿瘤强度的一阶统计特征; (ii) 反映肿瘤形状和大小 的特征; (iii) 描述肿瘤内部异质性的纹理特征; (iv) 小 波特征. 虽然有大量的研究致力于从医学影像中提取 更多的量化特征 ${ }^{[36,37]}$, 但非统一的算法定义以及非标 准化的图像处理流程对影像组学特征的可靠性产生了 一定的影响, 使得研究结果的再现性和可比性存在困 难. 为解决这一问题, Van Griethuysen等人 ${ }^{[38]}$ 开发了一 个开源的特征提取平台—Pyradiomics, 实现了影像 组学特征提取过程的标准化和可重复操作.

通常情况下, 为了更全面地刻画相关的临床问题, 影像组学分析会提取多个方面的高维特征. 但大量的 特征对于结果的分析并不一定是有利的, 需要足够的 样本量才能保证模型的有效性和鲁棒性, 而且大量的 特征中会存在干扰项、咒余项和不相干的特征. 所以, 为了避免出现过拟合 (overfitting) 现象, 提高模型的准
确性, 需要对提取的大量特征进行选择, 保留主要特 征. 特征选择的方法一般分为 3 类: (i) 过滤法: 根据 特征和分类标签的相关性选择特征. 基于过滤器的方 法一般是通过计算互信息、相关系数和单变量分析 (如Mann-Whitney U检验和卡方检验)等来实现的. (ii) 包装法: 根据特征预测效果评分选择特征, 通常与指定 的分类器相结合. 它能够选择预测能力最高的特征, 并 删除对预测贡献最小的特征, 直到选择了指定的特征 个数. (iii) 嵌入法: 根据模型训练过程中的权值系数 选择特征. 该方法的一个典型例子是最小绝对收缩和 选择算子(least absolute shrinkage and selection operator, LASSO)算法. 基于过滤器的方法相较于其他两种 方法具有计算时间短的优点, 但选择过程缺乏训练学 习, 预测精度往往较低. 因此, 在影像组学分析中, 基 于过滤器的特征选择方法常用于初步篎选.

\section{4 分析和建模}

影像组学的建模过程是通过机器学习技术实现从 
影像到临床目标预测的过程. 多数机器学习模型已经 在影像组学分析中显示出其有效性, 如逻辑回归(1ogistic regression)、最近邻(nearest neighbor)、支持向 量机(support vector machine, SVM)、随机森林(random forest)和人工神经网络(artificial neural networks) 等. 通常根据研究人员的偏好和经验选择建模方法, 但 不同的建模方法有其不同的局限性，如逻辑回归模型 对所选特征的独立性假设、贝叶斯网络模型对特征离 散化的要求、深度学习模型的网络配置依赖性. 研究 表明, 建模方法能够影响影像组学分析的预测结果 ${ }^{[39]}$. 因此，可以尝试进行多种建模方法来实现最优的模型 选择.

\section{5 质量评估}

未经验证的预测模型的临床应用价值是有限的, 完整的影像组学分析应该包含模型性能的评估和验 证. 预测模型的性能通常是根据其对相应临床目标的 识别能力和校正能力来进行评估的. 最常用的方法是 利用接收者工作特性(receiver operating characteristics, ROC)曲线和ROC曲线下面积(area under curve, AUC) 来评估模型的识别能力, 并量化模型的准确性、敏感 性和特异性; 而校正曲线可以评估模型结果和观察结 果间的一致性，衡量模型的整体表现. 所有的预测模 型都应进行内部验证，并在条件允许的情况下进行外 部验证(多中心验证), 因为外部验证的模型比内部验 证的模型更具可靠性.

\section{2 基于深度学习的影像组学}

深度学习是机器学习研究中的一个分支, 其在深 度神经网络(deep neural networks)的基础上发展起来, 创建复杂的神经结构来解决问题, 目前已经在影像组 学研究中得到了广泛的应用 ${ }^{[40-42]}$. 传统影像组学特征 侧重于从多个角度刻画肿瘤信息，而深度学习特征借 助深度学习强大的自学习能力根据临床标签从数据中 自动学习特征. 因此, 深度学习特征可以更加全面和深 层次地挖掘相关的影像信息.

目前, 影像组学研究中常用的深度学习模型主要 包括3种类型: 卷积神经网络(convolutional neural network, $\mathrm{CNN})^{[43]}$ 、生成对抗模型(generative adversarial networks, GAN) ${ }^{[44]}$ 和稀疏自编码器(sparse autoencoder,
$\mathrm{SAE})^{[45]}$. CNN通过卷积的方式将图像中每个像素与其 周围的点看作一个整体，这种方法把每个小的图片当 作特征进行学习，更加关注图像中不同点之间的关联 性. 它能够根据数据和临床目标定向地从图像中自动 学习有效特征, 不需要进行图像分割和提取人工特征. GAN由两个CNN构成, 一个作为生成器, 另一个作为 判别器, 通过对抗学习的方式来训练模型. 生成器捕捉 真实数据样本的潜在分布并生成新的数据样本; 判别 器用于判断输入的是一个真实数据还是生成数据. SAE是一种无监督学习方法, 通过计算自编码的输出 和原始输入的误差, 不断调整编码器的参数, 最终得 到预测模型. 它仅学习图像的稀疏表示, 尝试找出可 以反映图像的本质特征, 不与任何标签直接相关联. 3 个模型的相同之处是都不需要单独分割图像和提取特 征, 模型结构都包含卷积层和池化层.

\section{3 肝癌的术前诊断}

\section{1 病变类型鉴别}

临床中常用的肝癌影像学篮查方式包括超声造影 (contrast-enhanced ultrasound)、CT和MRI等, 在肝癌 的诊断及分期分级中起着重要的作用. HCC 作为最常 见的原发性肝癌, 是唯一不需要进一步病理证实即可 根据典型的特征增强模式来确诊的实体瘤. 然而，一 些非 HCC 病变(如小导管型肝内胆管癌和合并肝内胆 管癌等恶性肿瘤、肝血管瘤和肝腺瘤等局灶良性病 变)在常规影像学上与HCC表现相似, 当前的影像学方 法在这些病变的诊断中仍存在困难. 所以, 基于影像学 的肝癌早期准确诊断对临床决策和治疗具有重要意 义. Virmani等人 ${ }^{[46]}$ 利用超声影像提取了一阶统计特 征、纹理特征、傅里叶变换特征、小波变换特征和 Laws特征，并结合神经网络分类器，显著提高了肝脏 局灶病变(包括典型和非典型囊肿、血管瘤、转移瘤 和肝细胞癌)分类的准确率，最高达到 $95 \% . \mathrm{Li}^{\text {等人 }}{ }^{[47]}$ 基于Spair T2加权MRI提取纹理特征，结合4种分类器 (K近邻, KNN; 反向传播网络, BP-ANN; SVM; 逻辑回 归)识别肝血管瘤、肝转移瘤和HCC; 结果表明, 没有 某个纹理特征可以同时区分 3 种类型的肿瘤, 而对于每 两种肿瘤类别的鉴别，3个模型的错误率分别是 $11.7 \%$ (肝血管瘤和肝转移瘤)、9.6\%(肝转移瘤和 $\mathrm{HCC}$ ) 和 9.7\%(肝血管瘤和HCC), 说明基于Spair T2加权MRI的 
纹理特征可以作为此类病变诊断的辅助手段. Lewis等 人 $^{[14]}$ 利用弥散加权成像(diffusion weighted imaging)的 表观弥散系数(apparent diffusion coefficient, ADC)直方 图参数与肝脏影像报告和数据管理系统(liver imaging reporting and data system, LI-RADS)分类对HCC和其 他原发性肝癌(ICC, HCC-ICC)进行鉴别，结果表明融 合了性别、第 5 百分位 $\mathrm{ADC}(5 \% \mathrm{ADC})$ 和LI-RADS分类 的预测模型得到了最优的预测性能: HCC 与其他原发 瘤鉴别的AUC为 0.90, ICC 与 HCC-ICC鉴别的AUC为 0.89. Yao 等人 ${ }^{[13]}$ 利用多参数超声成像和深度学习技术 对 HCC进行诊断并预测临床行为, 研究共建立 5 个影像 组学模型：肿瘤良恶性鉴别模型的AUC为 $0.94(95 \%$ 置 信区间(confidence interval, CI): 0.88 0.98), 恶性肿瘤 类型 (HCC 和其他)鉴别模型的 $\mathrm{AUC}$ 为 $0.97(95 \% \mathrm{CI}$ : 0.93 0.99), 程序性死亡受体1(programmed cell death protein 1, PD-1)预测模型的 AUC 是 $0.97(95 \%$ CI: 0.89 0.98), Ki-67抗原预测模型的AUC为 $0.94(95 \% \mathrm{CI}$ : 0.87 0.97), 微血管侵犯(microvascular invasion, MVI) 的预测模型AUC为 $0.98(95 \%$ CI: 0.93 0.99). 说明基于 肿瘤多参数超声影像的影像组学分析有助于肝脏肿瘤 的诊断和临床预后的综合评价. 这些研究体现了机器 学习和深度学习在肝癌诊断中的价值, 促进了影像组 学的发展.

\section{2 肝癌MVI预测}

MVI是肝癌患者手术治疗后早期复发的重要独立 影响因子. 既往研究表明, 肝癌MVI出现提示较强的肿 瘤生物学侵袭性, 可使肝癌复发率增加 4.4 倍 ${ }^{[48]}$. 术前 进行肝癌MVI的准确评估和预测可以帮助医生及时调 整治疗策略(如扩大切除范围), 降低术后复发风险, 改 善患者预后，对肝癌患者的个体化精准治疗具有重要 的临床意义. 2017年, Bakr等人 ${ }^{[17]}$ 利用 $\mathrm{CT}$ 影像数据提 取了包括图像强度、肿瘤形状、纹理和边缘信息的定 量影像特征, 对肝癌患者MVI进行预测, 最终结果AUC 为 $0.76(95 \%$ CI: 0.58 0.94). 虽然该研究结果比较基础, 但是为利用影像组学技术探究MVI的潜在替代生物标 记物提供了研究依据. 此后, 许多研究团队针对这一临 床问题利用不同模态的医学影像进行了研究 ${ }^{[18-22]}$. Ma 等人 ${ }^{[20]}$ 基于术前的 $\mathrm{CT}$ 影像提取影像组学特征, 之后利 用LASSO回归方法选择最有效的预测特征并构建 MVI预测模型. 结果表明，基于门静脉期 CT 影像的预
测结果优于动脉期和延迟期的结果, 而且融合了门静 脉期影像组学标签和临床变量的模型在验证集中取得 最优的预测性能: AUC为 $0.80(95 \%$ CI: $0.67 \sim 0.93)$, 敏 感性为 0.90 , 特异性为 0.76 . 此外, Yang 等人 ${ }^{[22]}$ 基于术 前MRI和临床因子构建MVI的预测模型，在验证集中 的AUC为 $0.86(95 \% \mathrm{CI}: 0.75 \sim 0.97)$, 敏感性为 0.90 , 特 异性为 $0.81 . \mathrm{Xu}$ 等人 ${ }^{[21]}$ 基于术前的增强 $\mathrm{CT}$ 影像提取影 像组学特征, 结合肝癌患者的临床特征和定性影像特 征构建MVI预测模型，在验证集中的AUC为 $0.89(95 \%$ CI: $0.85 \sim 0.92)$, 每感性为 0.90 , 特异性为 0.79 ; 虽然决 策曲线分析表明影像组学标签并未增加净获益率(net benefit), 但单独的影像组学标签仍然取得了良好的预 测性能(AUC: 0.80 0.82). 这些研究为肝癌MVI的术前 评估和预测提供了一种新的方法.

\section{3 肝癌的病理分级}

研究表明, $\mathrm{HCC}$ 的病理等级是患者术后复发的影 响因子之一, 高级别的 HCC患者比低级别的HCC患者 具有更高的肝内复发率 ${ }^{[49,50]}$. 相对于低级别 HCC患者, 大多数的高级别 $\mathrm{HCC}$ 病人在手术切除时需要更大的 安全切缘, 以及更频繁的术后随访 ${ }^{[51,52]}$. 因此, 术前准 确地预测 $\mathrm{HCC}$ 患者的病理等级能够促进治疗策略的 选择. 针对该问题, $\mathrm{Wu}$ 等人 ${ }^{[15]}$ 基于 $\mathrm{T} 1$ 加权和 $\mathrm{T} 2$ 加权影 像提取影像组学特征, 利用LASSO回归算法选择最有 效的预测特征并构建预测 $\mathrm{HCC}$ 病理等级的影像组学 标签. 结果表明, 基于MRI的影像组学标签在高、低级 别的HCC患者中存在显著差异 $(P<0.05)$; 仅利用影像 组学特征的影像组学模型的预测性能优于仅基于临床 因素的预测模型(AUC: 0.74 vs. 0.60), 而融合影像组学 标签和临床因素的组合模型取得最优的预测性能, AUC为0.80(95\% CI: 0.65 0.90). 这表明医学影像可以 作为HCC患者病理等级的独立预测工具, 并可以提高 临床因素的预测性能.

\section{4 个性化治疗方案的制定}

HCC 患者常伴有肝功能不全. HCC患者的死亡不 仅可能是由于肿瘤负担, 还可能是因为肝功能恶化引 起的 ${ }^{[53]}$. 因此, 在肝癌治疗过程中必须考虑创伤性. 肝 切除术是肝癌患者的首选治疗方式, 可以完整地切除 肿瘤, 使肿瘤细胞无残留, 但是具有较大的创伤性; 而 
经动脉化疗栓塞(transarterial chemoembolization, TACE)虽然是微创的，但治疗后可能会残留肿瘤细胞. 随着技术手段的不断发展，肝切除术和TACE的适用 范围都在不断扩大，并出现了重叠的情况 ${ }^{[54-59]}$. 在欧 洲肝脏研究协会和美国肝脏研究协会的指南中，对于 “不可切除HCC”的定义就是模棱两可的 ${ }^{[60,61]}$. 最新的 美国国家综合癌症网络(National Comprehensive Cancer Network, NCCN)指南指出, 在适合进行TACE治疗 的病人中进行肝切除术，其效果是“有争议的”[62]. 因 此，应根据患者的情况为患者选择合适的治疗方式. 但是，目前临床中治疗方式的选择存在局限性，如医 生在治疗选择上的主观性以及缺乏有效的治疗验证. 因此, 需要引入一种新的工具为HCC患者无创地、客 观地选择肝切除术或是TACE治疗，从而帮助患者制 定个性化的治疗方案. 针对此问题, $\mathrm{Fu}$ 等人 ${ }^{[23]}$ 利用 5 家 医院共 520 个 HCC 患者的术前 $\mathrm{CT}$ 影像数据构建了一个 用于辅助肝切除术和TACE治疗选择的模型. 为了控制 基线数据的差异, 研究对患者进行了加权, 并以无进展 生存率(progression-free survival, PFS)为终点构建了 COX回归模型. 结果表明，预测模型具有良好的鉴别 和校正能力: 训练集和测试集中 3 年PFS的AUC分别是 0.80 和 0.75 ; 将该预测模型与其他 4 个最先进的模型进 行比较, 也得到了更高的预测精度. 研究最后构建了 诺模图, 从而可以根据不同得分选择相应治疗方式(肝 切除术或TACE). 根据得分阈值(阈值 $=-5.00$ ) 将患者进 行分类, 当得分小于等于 -5.00 时, 肝切除术可以得到 比TACE更长的PFS(测试集中风险比(hazard ratio, HR) 为 $0.52,95 \%$ CI: 0.29 0.93, $P$ 值为 0.026 ), 所以肝切除术 被建议为更好的治疗选择; 当得分大于-5.00时，肝切 除术与 TACE两种治疗间的PFS无统计差异(测试集中 HR为 $1.14,95 \%$ CI: $0.69 \sim 1.85, P$ 值为 0.614$)$, 但是 TACE 的创伤性更小, 因此可以作为更好的治疗选择.

\section{5 复发及生存期预测}

\section{1 早期复发及无复发生存期}

根据巴塞罗那分期(Barcelona Clinic Liver Cancer, BCLC)系统，最早期和早期的肝癌患者的治疗方式包 括肝切除术、肝移植术和射频消融术，而且肝切除术 是非肝硬化患者以及肝功能良好的肝硬化患者的首选 治疗方法 ${ }^{[63-65]}$. 然而, 治疗后的复发仍然是影响治疗
效果的一个主要挑战. 术前识别高危复发患者能够指 导手术管理、术后监测和治疗干预.

对于肝切除术患者，从切除到复发的时间间隔是 一个独立的预后因子，而且早期(1年内)复发患者的预 后比晚期(1年以上)的预后更差 ${ }^{[66,67]}$.2017年，Zhou等 人 $^{[24]}$ 利用术前动脉期和门静脉期 CT 影像分别提取了 300 个直方图和灰度共生矩阵特征, 之后利用 LASSO 回归方法选择最有效的预测特征并构建早期复发预测 模型. 最终融合临床变量和影像组学标签的组合模型 取得最优的预测性能， A U C 为 $0.84(95 \% \mathrm{CI}$ : $0.78 \sim 0.88$ ), 敏感性为 0.82 , 特异性为 0.71 .2018 年, Hui 等人 ${ }^{[25]}$ 利用术前的MRI提取了 290 个纹理参数预测 HCC 患者手术切除后 730 天的复发情况, 结果表明, 动 态增强MRI平衡期影像的和方差 $\mathrm{S}(4,0)$ 取得了最优的 预测结果, 准确率为 0.84 . 所以, 基于术前的 CT或MRI 的影像组学分析可以作为一种新的工具预测肝癌早期 复发。

肝移植是终末期肝癌患者最有效的治疗方式，被 推荐用于临床证实的门静脉高压症患者和符合米兰标 准的早期肝癌患者，5年的无病生存率高达 $60 \%$ $\sim 80 \%{ }^{[68,69]}$. 但是在 $20 \%$ 接受肝移植的患者中发现了 MVI导致的早期复发，可使 5 年生存率从 $80 \%$ 降至 $40 \%{ }^{[69 \sim 71]}$. 复发是影响肝移植术后疗效的主要因素 ${ }^{[72]}$. 因此，对肝移植患者的复发情况进行预测可以直接影 响器官分配、手术技术和预后等.Guo等人 ${ }^{[26]}$ 基于术 前的肝动脉期 CT 图像提取影像组学特征, 利用LASSO-COX回归选择最优的预测特征构建 COX回归模 型; 融合影像组学标签和临床因素的组合模型显示出 最优的预测性能，模型在测试集中的一致性指数(concordance index, C-index)为0.79(95\% CI: $0.62 \sim 0.96)$, 校正曲线亦显示出较好的一致性 $(P=0.164)$. 研究为术 前无创地预测肝移植患者的复发情况提供了一种有效 的工具, 具有重要的临床意义.

肝功能不全、伴发疾病或肝源不足等，使得肝切 除术和肝移植的应用受到限制. 因此，局部手术技术， 如射频消融、微波消融和冷冻消融，被引入到临床中. 消融后早期复发(消融治疗后2年内出现新发病灶)是 导致患者治疗后死亡的主要因素之一 ${ }^{[73 \sim 77]}$. 研究表明, 晚期复发的患者生存率很可能优于早期复发患 者 ${ }^{[76,77]}$. 肝癌患者消融治疗后复发的早期发现和及时 治疗可以改善预后 ${ }^{[78]}$. 但目前临床中还没有有效的工 
具能够预测消融后复发. Yuan等人 ${ }^{[27]}$ 利用 184 例 HCC 患者的术前CT影像数据, 以无复发生存期(recurrence free survival)为终点, 研究CT影像数据与消融后复发 的关系. 研究在三期(动脉期、静脉期和实质期)增强 CT影像中提取影像组学特征, 先利用最大相关-最小 穴余算法(maximum relevance-minimum redundancy) 进行初步的特征选择, 再利用LASSO-COX回归算法 分别构建 3 个时期的影像组学模型, 最后融合 3 个时期 的影像组学标签构建最终的影像组学模型. 在所有的 4 个影像组学模型中, 静脉期影像组学模型在验证集 的表现最优, C-index为0.74(95\% CI: 0.63 0.84). 将静 脉期影像组学标签和临床病理因素相结合, 研究得到 了最优的预测性能, 验证集的C-index为 $0.76(95 \% \mathrm{CI}$ : $0.65 \sim 0.86$ ), 比单纯利用临床变量的临床模型 (C-in$\mathrm{dex}=0.56,95 \% \mathrm{CI}: 0.47 \sim 0.64)$ 有显著提高. 研究表明, 结合临床病理因素和影像组学特征的模型, 能够用于 $\mathrm{HCC}$ 根治消融后的复发预测.

\section{2 总生存期}

尽管 HCC 患者肝切除术后有高达 $70 \%$ 的存活

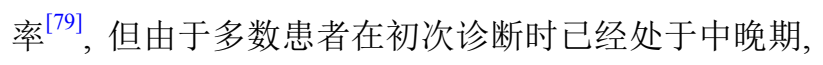
适合切除的患者不足 $30 \%$. 此外, 研究表明, TACE对于 治疗中晚期患者是有效的 ${ }^{[80,81]}$, 但是TACE治疗患者的 长期生存并不令人满意 ${ }^{[82 ~ 84]}$. 基于这些临床反应, 开 发和验证一种可以在治疗前预测患者生存率的可能性 工具, 以便更好地个性化治疗是非常重要的. Zheng等 人 $^{[28]}$ 基于术前的 $\mathrm{CT}$ 影像数据提取纹理特征, 利用 LASSO回归算法选择最有效的影像组学特征, 并结合 临床病理因素构建了COX回归模型以预测 HCC 患者 的总生存率(overall survival, OS), 研究表明, 影像组学 标签与 $\mathrm{OS}$ 显著相关 $(\mathrm{HR}=3.24,95 \% \mathrm{CI}: 1.42 \sim 7.41$, $P=0.005)$, 而且影像组学标签和临床病理因素融合模 型取得了最优的预测性能 $(\mathrm{C}-$-index $=0.71,95 \% \mathrm{CI}$ :
0.60 0.82). Kim等人 ${ }^{[29]}$ 基于 TACE治疗前的 CT影像数 据提取影像组学特征, 利用LASSO-COX回归算法选 择最优的预测特征, 并结合影像组学特征和临床因素 构建组合COX回归模型, 研究表明, 与临床模型和影 像组学模型相比, 组合模型能够更好地预测HCC患者 TACE治疗后生存率(HR=19.88, 95\% CI: 6.37 92.02, $P<0.001$ ). 此外, Blanc-Durand等人 ${ }^{[31]}$ 基于肝动脉 $90 \mathrm{Y}$ 放射性栓塞(transarterial radioembolization using Yttrium-90, 90Y-TARE)治疗前的PET影像数据, 对HCC 患者PFS和OS进行预测, 并利用LASSO-COX回归算 法构建预后标签, 研究表明, 基于PET的影像组学标签 与PFS(HR=120, 95\% CI: 3.98 3625, $P=0.006$ ) 和OS (HR=16.1, 95\% CI: 2.94 88.3, $P=0.001)$ 显著相关, 可以 作为HCC 患者PFS和OS的独立预测因子. 这些研究表 明, HCC患者治疗前的影像数据可以用于预测生存率, 为患者的个性化治疗提供帮助.

\section{6 总结}

目前, 影像组学已经形成了一套完整的理论体系 和技术框架, 其在肿瘤的诊断、治疗策略的选择、疗 效的预测和预后评估等方面显示出巨大的潜力. 除原 发性肝癌中的研究, 影像组学在转移性肝癌以及其他 肝病中也得到了很好地应用 ${ }^{[85 ~ 89]}$. 影像组学正在弥合 个性化管理和计算机辅助预测之间的鸿沟. 随着更多 更新的治疗方式的出现, 如图像引导的放疗、呼吸适 应放疗、免疫治疗等, 影像组学将为个性化治疗策略 的实施铺平道路. 除了这些治疗方式外, 影像组学最 重要的作用是有希望将实验结果应用到生命科学的基 础医学当中, 如预测 $\mathrm{HCC}$ 和表观遗传学、引起 $\mathrm{HCC}$ 和 ICC不同病变的微环境等. 随着人工智能技术的发展, 影像组学将临床影像成像技术推向了个性化治疗策略 制定的重要位置, 成为了肝病患者的重要临床标志物.

致谢感谢中国科学院自动化研究所多模态生物医学成像实验平台、北京航空航天大学大数据精准医疗高精尖创新 中心、西安电子科技大学生命科学技术学院分子与神经影像教育部工程研究中心对本文的大力支持和帮助.

\section{参考文献}

1 Hricak H. Oncologic imaging: a guiding hand of personalized cancer care. Radiology, 2011, 259: 633-640

2 Sharma B, Martin A, Stanway S, et al. Imaging in oncology—Over a century of advances. Nat Rev Clin Oncol, 2012, 9: 728-737 
3 Hood L, Friend S H. Predictive, personalized, preventive, participatory (P4) cancer medicine. Nat Rev Clin Oncol, 2011, 8: 184-187

4 Lambin P, Rios-Velazquez E, Leijenaar R, et al. Radiomics: Extracting more information from medical images using advanced feature analysis. Eur J Cancer, 2012, 48: 441-446

5 Aerts H J W L, Velazquez E R, Leijenaar R T H, et al. Decoding tumour phenotype by noninvasive imaging using a quantitative radiomics approach. Nat Commun, 2014, 5: 4006

6 Torre L A, Bray F, Siegel R L, et al. Global cancer statistics, 2012. CA A Cancer J Clin, 2015, 65: 87-108

7 Chen W, Zheng R, Baade P D, et al. Cancer statistics in China, 2015. CA A Cancer J Clin, 2016, 66: 115-132

8 Lin D C, Mayakonda A, Dinh H Q, et al. Genomic and epigenomic heterogeneity of hepatocellular carcinoma. Cancer Res, 2017, 77: 2255-2265

9 Lu L C, Hsu C H, Hsu C, et al. Tumor heterogeneity in hepatocellular carcinoma: facing the challenges. Liver Cancer, 2016, 5: 128-138

10 Segal E, Sirlin C B, Ooi C, et al. Decoding global gene expression programs in liver cancer by noninvasive imaging. Nat Biotechnol, 2007, 25: $675-680$

11 Kuo M D, Gollub J, Sirlin C B, et al. Radiogenomic analysis to identify imaging phenotypes associated with drug response gene expression programs in hepatocellular carcinoma. J Vasc Interv Rad, 2007, 18: 821-830

12 Miura T, Ban D, Tanaka S, et al. Distinct clinicopathological phenotype of hepatocellular carcinoma with ethoxybenzyl-magnetic resonance imaging hyperintensity: association with gene expression signature. Am J Surg, 2015, 210: 561-569

13 Yao Z, Dong Y, Wu G, et al. Preoperative diagnosis and prediction of hepatocellular carcinoma: Radiomics analysis based on multi-modal ultrasound images. BMC Cancer, 2018, 18: 1089

14 Lewis S, Peti S, Hectors S J, et al. Volumetric quantitative histogram analysis using diffusion-weighted magnetic resonance imaging to differentiate HCC from other primary liver cancers. Abdom Radiol, 2019, 44: 912-922

$15 \mathrm{Wu}$ M, Tan H, Gao F, et al. Predicting the grade of hepatocellular carcinoma based on non-contrast-enhanced MRI radiomics signature. Eur Radiol, 2019, 29: 2802-2811

16 Peng J, Qi X, Zhang Q, et al. A radiomics nomogram for preoperatively predicting prognosis of patients in hepatocellular carcinoma. Transl Cancer Res, 2018, 7: 936-946

17 Bakr S, Echegaray S, Shah R, et al. Noninvasive radiomics signature based on quantitative analysis of computed tomography images as a surrogate for microvascular invasion in hepatocellular carcinoma: a pilot study. J Med Imag, 2017, 4: 1

$18 \mathrm{Hu} \mathrm{H} \mathrm{T,} \mathrm{Wang} \mathrm{Z,} \mathrm{Huang} \mathrm{X} \mathrm{W,} \mathrm{et} \mathrm{al.} \mathrm{Ultrasound-based} \mathrm{radiomics} \mathrm{score:} \mathrm{a} \mathrm{potential} \mathrm{biomarker} \mathrm{for} \mathrm{the} \mathrm{prediction} \mathrm{of} \mathrm{microvascular} \mathrm{invasion} \mathrm{in}$ hepatocellular carcinoma. Eur Radiol, 2019, 29: 2890-2901

19 Feng S T, Jia Y, Liao B, et al. Preoperative prediction of microvascular invasion in hepatocellular cancer: a radiomics model using Gd-EOBDTPA-enhanced MRI. Eur Radiol, 2019, 29: 4648-4659

20 Ma X, Wei J, Gu D, et al. Preoperative radiomics nomogram for microvascular invasion prediction in hepatocellular carcinoma using contrastenhanced CT. Eur Radiol, 2019, 29: 3595-3605

21 Xu X, Zhang H L, Liu Q P, et al. Radiomic analysis of contrast-enhanced CT predicts microvascular invasion and outcome in hepatocellular carcinoma. J Hepatol, 2019, 70: 1133-1144

22 Yang L, Gu D, Wei J, et al. A radiomics nomogram for preoperative prediction of microvascular invasion in hepatocellular carcinoma. Liver Cancer, 2019, 8: 373-386

23 Fu S, Wei J, Zhang J, et al. Selection between liver resection versus transarterial chemoembolization in hepatocellular carcinoma. Clin Transl Gastroenterol, 2019, 10: e00070

24 Zhou Y, He L, Huang Y, et al. CT-based radiomics signature: a potential biomarker for preoperative prediction of early recurrence in hepatocellular carcinoma. Abdom Radiol, 2017, 42: 1695-1704

25 Hui T C H, Chuah T K, Low H M, et al. Predicting early recurrence of hepatocellular carcinoma with texture analysis of preoperative MRI: a radiomics study. Clin Rad, 2018, 73: 1056.e11-1056.e16

26 Guo D, Gu D, Wang H, et al. Radiomics analysis enables recurrence prediction for hepatocellular carcinoma after liver transplantation. Eur J Rad, 2019, 117: 33-40

27 Yuan C, Wang Z, Gu D, et al. Prediction early recurrence of hepatocellular carcinoma eligible for curative ablation using a radiomics nomogram. Cancer Imag, 2019, 19: 21

28 Zheng B H, Liu L Z, Zhang Z Z, et al. Radiomics score: a potential prognostic imaging feature for postoperative survival of solitary HCC 
patients. BMC Cancer, 2018, 18: 1148

29 Kim J, Choi S J, Lee S H, et al. Predicting survival using pretreatment CT for patients with hepatocellular carcinoma treated with transarterial chemoembolization: Comparison of models using radiomics. Am J Roentgenol, 2018, 211: 1026-1034

30 Cozzi L, Dinapoli N, Fogliata A, et al. Radiomics based analysis to predict local control and survival in hepatocellular carcinoma patients treated with volumetric modulated arc therapy. BMC Cancer, 2017, 17: 829

31 Blanc-Durand P, Van Der Gucht A, Jreige M, et al. Signature of survival: a ${ }^{18}$ F-FDG PET based whole-liver radiomic analysis predicts survival after ${ }^{90}$ Y-TARE for hepatocellular carcinoma. Oncotarget, 2018, 9: 4549-4558

32 Gillies R J, Kinahan P E, Hricak H. Radiomics: Images are more than pictures, they are data. Radiology, 2016, 278: 563-577

33 Yip S S F, Aerts H J W L. Applications and limitations of radiomics. Phys Med Biol, 2016, 61: R150-R166

34 Larue R T H M, Defraene G, De Ruysscher D, et al. Quantitative radiomics studies for tissue characterization: a review of technology and methodological procedures. BJR, 2017, 90: 20160665

35 Grootjans W, Tixier F, van der Vos C S, et al. The impact of optimal respiratory gating and image noise on evaluation of intra-tumor heterogeneity on ${ }^{18}$ F-FDG PET imaging of lung cancer. J Nucl Med, 2016, 57: 1692-1698

36 Kumar V, Gu Y, Basu S, et al. Radiomics: the process and the challenges. Magn Reson Imag, 2012, 30: 1234-1248

37 Huang Y, Liu Z, He L, et al. Radiomics signature: A potential biomarker for the prediction of disease-free survival in early-stage (I or II) nonsmall cell lung cancer. Radiology, 2016, 281: 947-957

38 van Griethuysen J J M, Fedorov A, Parmar C, et al. Computational radiomics system to decode the radiographic phenotype. Cancer Res, 2017, 77: e104-e107

39 Parmar C, Grossmann P, Bussink J, et al. Machine learning methods for quantitative radiomic biomarkers. Sci Rep, 2015, 5: 13087

40 Wang S, Zhou M, Liu Z, et al. Central focused convolutional neural networks: developing a data-driven model for lung nodule segmentation. Med Image Anal, 2017, 40: 172-183

41 Esteva A, Kuprel B, Novoa R A, et al. Dermatologist-level classification of skin cancer with deep neural networks. Nature, 2017, 542: 115-118

42 Kermany D S, Goldbaum M, Cai W, et al. Identifying medical diagnoses and treatable diseases by image-based deep learning. Cell, 2018, 172: 1122-1131.e9

43 LeCun Y, Bengio Y, Hinton G. Deep learning. Nature, 2015, 521: 436-444

44 Goodfellow I J, Pouget-Abadie J, Mirza M, et al. Generative adversarial nets. In: 28th Annual Conference on Neural Information Processing Systems 2014, NIPS 2014, December 8, 2014-December 13, 2014, January. Montreal, 2014. La Jolla: Neural Information Processing Systems Foundation, 2015. 2672-2680

45 Lee H, Grosse R, Ranganath R, et al. Unsupervised learning of hierarchical representations with convolutional deep belief networks. Commun ACM, 2011, 54: 95-103

46 Virmani J, Kumar V, Kalra N, et al. Neural network ensemble based cad system for focal liver lesions from B-mode ultrasound. J Digit Imag, 2014, 27: 520-537

47 Li Z, Mao Y, Huang W, et al. Texture-based classification of different single liver lesion based on spair T2W MRI images. BMC Med Imag, 2017, 17: 42

48 Miyata R, Tanimoto A, Wakabayashi G, et al. Accuracy of preoperative prediction of microinvasion of portal vein in hepatocellular carcinoma using superparamagnetic iron oxide-enhanced magnetic resonance imaging and computed tomography during hepatic angiography. J Gastroenterol, 2006, 41: 987-995

49 Sasaki A, Kai S, Iwashita Y, et al. Microsatellite distribution and indication for locoregional therapy in small hepatocellular carcinoma. Cancer, 2005, 103: 299-306

$50 \mathrm{Ng}$ I O L, Lai E C S, Fan S T, et al. Prognostic significance of pathologic features of hepatocellular carcinoma a multivariate analysis of 278 patients. Cancer, 1995, 76: 2443-2448

51 Okusaka T, Okada S, Ueno H, et al. Satellite lesions in patients with small hepatocellular carcinoma with reference to clinicopathologic features. Cancer, 2002, 95: 1931-1937

52 Bruix J, Sherman M. Management of hepatocellular carcinoma. Hepatology, 2005, 42: 1208-1236

53 Dhir M, Melin A A, Douaiher J, et al. A review and update of treatment options and controversies in the management of hepatocellular carcinoma. Ann Surg, 2016, 263: 1112-1125 
54 Vitale A, Burra P, Frigo A C, et al. Survival benefit of liver resection for patients with hepatocellular carcinoma across different Barcelona Clinic Liver Cancer stages: A multicentre study. J Hepatol, 2015, 62: 617-624

55 Yin L, Li H, Li A J, et al. Partial hepatectomy vs. transcatheter arterial chemoembolization for resectable multiple hepatocellular carcinoma beyond Milan criteria: A RCT. J Hepatol, 2014, 61: 82-88

56 Kokudo T, Hasegawa K, Matsuyama Y, et al. Liver resection for hepatocellular carcinoma associated with hepatic vein invasion: A Japanese nationwide survey. Hepatol, 2017, 66: 510-517

57 Zhu K, Chen J, Lai L, et al. Hepatocellular carcinoma with portal vein tumor thrombus: Treatment with transarterial chemoembolization combined with sorafenib-A retrospective controlled study. Radiology, 2014, 272: 284-293

58 Yang T, Lin C, Zhai J, et al. Surgical resection for advanced hepatocellular carcinoma according to Barcelona Clinic Liver Cancer (BCLC) staging. J Cancer Res Clin Oncol, 2012, 138: 1121-1129

59 Pinter M, Hucke F, Graziadei I, et al. Advanced-stage hepatocellular carcinoma: Transarterial chemoembolization versus sorafenib. Radiology, 2012, 263: 590-599

60 Galle P R, Forner A, Llovet J M, et al. EASL clinical practice guidelines: Management of hepatocellular carcinoma. J Hepatol, 2018, 69: 182-236

61 Marrero J A, Kulik L M, Sirlin C B, et al. Diagnosis, staging, and management of hepatocellular carcinoma: 2018 practice guidance by the American association for the study of liver diseases. Hepatology, 2018, 68: 723-750

62 National Comprehensive Cancer Network. Hepatobiliary Cancers (Version 3. 2019)

63 Clavien P A, Lesurtel M, Bossuyt P M, et al. Recommendations for liver transplantation for hepatocellular carcinoma: an international consensus conference report. Lancet Oncol, 2012, 13: e11-e22

64 Bruix J, Reig M, Sherman M. Evidence-based diagnosis, staging, and treatment of patients with hepatocellular carcinoma. Gastroenterology, 2016, 150: 835-853

65 Sangiovanni A, Colombo M. Treatment of hepatocellular carcinoma: beyond international guidelines. Liver Int, 2016, 36: 124-129

66 Poon R T P, Fan S T, Ng I O L, et al. Different risk factors and prognosis for early and late intrahepatic recurrence after resection of hepatocellular carcinoma. Cancer, 2000, 89: 500-507

67 Shimada M, Takenaka K, Gion T, et al. Prognosis of recurrent hepatocellular carcinoma: a 10-year surgical experience in Japan. Gastroenterology, 1996, 111: 720-726

68 Fong Y, Sun R L, Jarnagin W, et al. An analysis of 412 cases of hepatocellular carcinoma at a Western center. Ann Surgery, 1999, 229: 790-799

69 Lim K C, Chow P K H, Allen J C, et al. Microvascular invasion is a better predictor of tumor recurrence and overall survival following surgical resection for hepatocellular carcinoma compared to the Milan criteria. Ann Surg, 2011, 254: 108-113

70 Renzulli M, Buonfiglioli F, Conti F, et al. Imaging features of microvascular invasion in hepatocellular carcinoma developed after direct-acting antiviral therapy in HCV-related cirrhosis. Eur Radiol, 2018, 28: 506-513

71 Kim M J, Lee M, Choi J Y, et al. Imaging features of small hepatocellular carcinomas with microvascular invasion on gadoxetic acid-enhanced MR imaging. Eur J Rad, 2012, 81: 2507-2512

72 Taketomi A, Fukuhara T, Morita K, et al. Improved results of a surgical resection for the recurrence of hepatocellular carcinoma after living donor liver transplantation. Ann Surg Oncol, 2010, 17: 2283-2289

73 Pompili M, Saviano A, de Matthaeis N, et al. Long-term effectiveness of resection and radiofrequency ablation for single hepatocellular carcinoma $3 \mathrm{~cm}$. Results of a multicenter Italian survey. J Hepatol, 2013, 59: 89-97

74 Heimbach J K. Overview of the updated AASLD guidelines for the management of HCC. Gastroenterol Hepatol (NY). 2017, 13: 751-753 51: $890-897$

77 Portolani N, Coniglio A, Ghidoni S, et al. Early and late recurrence after liver resection for hepatocellular carcinoma. Ann Surgery, 2006, 243: $229-235$

78 Benson 3rd A B, D’Angelica M I, Abbott D E, et al. NCCN guidelines insights: Hepatobiliary cancers, version 1.2017. J Natl Compr Canc Netw, 2017, 15: 563-573

79 El-Serag H B, Mason A C. Rising incidence of hepatocellular carcinoma in the United States. N Engl J Med, 1999, 340: 745-750

80 Bruix J, Sala M, Llovet J M. Chemoembolization for hepatocellular carcinoma. Gastroenterology, 2004, 127: S179-S188 
81 Llovet J M, Real M I, Montaña X, et al. Arterial embolisation or chemoembolisation versus symptomatic treatment in patients with unresectable hepatocellular carcinoma: a randomised controlled trial. Lancet, 2002, 359: 1734-1739

82 Lo C M, Ngan H, Tso W K, et al. Randomized controlled trial of transarterial lipiodol chemoembolization for unresectable hepatocellular carcinoma. Hepatology, 2002, 35: 1164-1171

83 Cammà C, Schepis F, Orlando A, et al. Transarterial chemoembolization for unresectable hepatocellular carcinoma: meta-analysis of randomized controlled trials. Radiology, 2002, 224: 47-54

84 Mabed M, Esmaeel M, El-Khodary T, et al. A randomized controlled trial of transcatheter arterial chemoembolization with lipiodol, doxorubicin and cisplatin versus intravenous doxorubicin for patients with unresectable hepatocellular carcinoma. Eur J Cancer Care, 2009, 18: 492-499

85 Wang K, Lu X, Zhou H, et al. Deep learning Radiomics of shear wave elastography significantly improved diagnostic performance for assessing liver fibrosis in chronic hepatitis B: a prospective multicentre study. Gut, 2019, 68: 729-741

86 Naganawa S, Enooku K, Tateishi R, et al. Imaging prediction of nonalcoholic steatohepatitis using computed tomography texture analysis. Eur Radiol, 2018, 28: 3050-3058

87 Klaassen R, Larue R T H M, Mearadji B, et al. Feasibility of CT radiomics to predict treatment response of individual liver metastases in esophagogastric cancer patients. PLoS ONE, 2018, 13: e0207362

88 Dohan A, Gallix B, Guiu B, et al. Early evaluation using a radiomic signature of unresectable hepatic metastases to predict outcome in patients with colorectal cancer treated with FOLFIRI and bevacizumab. Gut, 2020, 69: 531-539

89 Rahmim A, Bak-Fredslund K P, Ashrafinia S, et al. Prognostic modeling for patients with colorectal liver metastases incorporating FDG PET radiomic features. Eur J Rad, 2019, 113: 101-109 


\title{
Research progress and clinical application of radiomics in hepatocellular carcinoma
}

\author{
HAN YuQi ${ }^{1,2}$, WEI JingWei ${ }^{2}, J_{A N G}$ HanYu $^{3}$, NIU Meng ${ }^{4}$, LIU Bing ${ }^{5}$, FU FangFang ${ }^{6}$, \\ GU DongSheng ${ }^{2}$, HAO XiaoHan ${ }^{2,7}$, LI HongJun ${ }^{8}$, SONG Bin ${ }^{3} \&$ TIAN Jie ${ }^{1,2,9,10,11}$ \\ 1 School of Life Science and Technology, Xidian University, Xi'an, 710126, China; \\ 2 CAS Key Laboratory of Molecular Imaging, Institute of Automation, Chinese Academy of Sciences, Beijing 100190, China; \\ 3 Department of Radiology, West China Hospital, Sichuan University, Chengdu 610041, China; \\ 4 Department of Interventional Radiology, The First Affiliated Hospital of China Medical University, Shenyang 110000, China; \\ 5 Department of Hepatobiliary Surgery, The First Medical Center of Chinese PLA General Hospital, Beijing 100853, China; \\ 6 Department of Medical Imaging, Henan Provincial People's Hospital, People's Hospital of Zhengzhou University, Zhengzhou 450003, China; \\ 7 Center of Biomedical Engineering, University of Science and Technology of China, Hefei 230027, China; \\ 8 Department of Radiology, Beijing YouAn Hospital, Capital Medicine University, Beijng 100069, China; \\ 9 Beijing Key Laboratory of Molecular Imaging, Beijing 100190, China; \\ 10 The State Key Laboratory of Management and Control for Complex Systems, Institute of Automation, Chinese Academy of Sciences, Beijing \\ 100190, China: \\ 11 Beijing Advanced Innovation Center for Big Data-Based Precision Medicine, School of Medicine, Beihang University, Beijing 100191, China \\ Medical image has been widely used in clinical application to aid decision making. It holds information related to tumor heterogeneity \\ that cannot be easily recognized. In radiomics analysis, medical images are converted into mineable high-dimensional quantitative \\ features by computer technology. Combining with related clinical problems, radiomics can provide help for prognosis and clinical \\ decision making. The emergence of radiomics represents the transformation in the evaluation of medical imaging from a descriptive \\ and qualitative discipline to a predictive and quantitative discipline. Hepatocellular carcinoma is one of the most common malignant \\ tumors in China with high morbidity and mortality. Radiomics has been used in diagnosis, treatment selection and efficacy evaluation \\ of hepatocellular carcinoma. Based on the basic principles and techniques of radiomics, this paper reviews the reaches in diagnosis, \\ treatment selection and efficacy evaluation of hepatocellular carcinoma.
}

hepatocellular carcinoma, radiomics, diagnosis, treatment selection, efficacy evaluation

doi: $10.1360 / \mathrm{SSV}-2019-0230$ 